\title{
Is distance to the nearest registered public automated defibrillator associated with the probability of bystander shock for victims of out-of-hospital cardiac arrest?
}

Joel Neves Briard BSc ${ }^{1}$, Luc de Montigny $\mathrm{PhD}^{2,3}$, Dave Ross $\mathrm{MD}^{2,4}$, François de Champlain MD FRCPC DABEM ${ }^{2,5}$, Eli Segal MD FRCPC FACEP $2,4,6$

This article has been published in a revised form in Prehospital and Disaster Medicine: https://doi.org/10.1017/S1049023X18000080

This version is free to view and download for private research and study only. Not for redistribution, re-sale or use in derivative works. (C) Cambridge University Press.

Submitted: 26 June 2017. Accepted: 24 August 2017.

\section{Affiliations :}

1 Université de Montréal Medical School, Quebec, Canada

2 Corporation d'Urgences-santé, Quebec, Canada

3 Department of Epidemiology, Biostatistics and Occupational Health, McGill University, Quebec, Canada

4 Department of Prehospital Medicine, Hôpital du Sacré-Cœur de Montréal, Quebec, Canada

5 Department of Emergency Medicine, McGill University Health Centre, Quebec, Canada

6 Department of Emergency Medicine, Jewish General Hospital, Quebec, Canada

Citation: Neves Briard J, de Montigny L, Ross D, de Champlain F, Segal E. Is distance to the nearest registered public automated defibrillator associated with the probability of bystander shock for victims of out-of-hospital cardiac arrest? Prehosp Disaster Med. 2018;33(2):153-159.

Short Title: Distance to Public AED and Shock Probability

Abstract (words): 256

Figures: 4
Text (words): 3157

Tables: 3

\section{Corresponding author information:}

\section{Eli Segal MD}

Research Module, Urgences-santé

6700 Rue Jarry East, 3rd Floor

Montreal, Quebec, Canada H1P 0A4

Email: eli.segal@mcgill.ca 


\title{
ORIGINAL RESEARCH
}

\section{Is Distance to the Nearest Registered Public Automated \\ Defibrillator Associated with the Probability of Bystander Shock for Victims of Out-of-Hospital Cardiac Arrest?}

\author{
Abbreviations \\ ACLS: $\quad$ Advanced cardiovascular life support \\ AED: $\quad$ Automated external defibrillator \\ aOR: $\quad$ Adjusted odds ratio \\ BLS: $\quad$ Basic life support \\ CI: $\quad$ Confidence interval \\ CPR: $\quad$ Cardiopulmonary resuscitation \\ EMS: $\quad$ Emergency medical services \\ OHCA: Out-of-hospital cardiac arrest \\ PAED: $\quad$ Public automated external defibrillator \\ ROSC: $\quad$ Return of spontaneous circulation
}

\begin{abstract}
Introduction: Rapid access to defibrillation is a key element in the management of out-ofhospital cardiac arrests (OHCA). Public automated external defibrillators (PAED) are becoming increasingly available, but little information exists regarding the relation between the proximity to the arrest and their usage in urban areas.
\end{abstract}


Methods: This study is a retrospective, observational, cross-sectional analysis of non-traumatic OHCA during a 24-month period in the greater Montreal area (Quebec, Canada). Using logistic regression, bystander shock odds are described with regards to distance from the OHCA scene to the nearest PAED, adjusted for prehospital care arrival delay and time of day, and stratifying for type of location.

Results: Out of a total of 2443 OHCA victims identified, 77 (3.2\%) received bystander PAED shock, $622(25.5 \%)$ occurred out-of-home, and $743(30.4 \%)$ occurred during business hours. When controlling for time (business hours vs. other hours) and mimimum response delay for prehospital care arrival, a marginal negative association was found between bystander shock and distance to the nearest PAED in logged meters $(\mathrm{aOR}=0.80, \mathrm{CI}: 0.64-0.99)$ for out-of-home cardiac arrests. No significant association was found between distance and bystander shock for at-home arrests. Out-of-home victims had significantly higher odds of receiving bystander shock up to 175 meters of distance to a PAED inclusively ( $\mathrm{aOR}=2.52, \mathrm{CI}: 1.07-5.89)$.

Conclusion: For out-of-home cardiac arrests, proximity to a PAED was associated with bystander shock in the greater Montreal area. Strategies aiming to increase accessibility and use of these life-saving devices could further expand this advantage by assisting bystanders in rapidly locating nearby PAED.

\section{Introduction}

Out-of-hospital cardiac arrests (OHCA) are an important public health issue in North America. Indeed, more than 400,000 OHCA occur annually, and at most $10.4 \%$ of victims survive to hospital discharge (1-4). 
In the minutes following OHCA onset, citizen bystanders have the ability to greatly influence the victim's chance of survival. Early bystander cardiopulmonary resuscitation (CPR) and automated external defibrillator (AED) use have been identified as two major factors in predicting OHCA survival (5-8). Such interventions have been shown to be extremely timesensitive: for every minute that elapses between the onset of OHCA and defibrillation with an AED is associated with a 7 to $10 \%$ decrease in survival probability $(8,9)$. Furthermore, the chances of surviving OHCA if more than 12 minutes have elapsed without defibrillation are less than $5 \%(9,10)$.

Bystanders employing an AED on an OHCA victim before the arrival of first responders increase the individual's chances of survival. While performing CPR, shocking with an AED doubles the victim's chances at survival $(11,12)$. Providing an AED shock even without providing CPR has also been shown to increase OHCA survival rates (13).

Unfortunately, in Canada, bystander shock preceding the arrival of first responders appears to be a rare event. In Southern Ontario for instance, bystander shock using a PAED only occurs in $5.6 \%$ of OHCA (14). In response to this issue, communities have invested significant resources in increasing the availability of AED in public spaces (public AED; PAED), such as in schools, residences, sports complexes and commercial buildings. However, when responding to an OHCA, bystanders are typically unaware of PAEDs' locations in the vicinity. In order to maximize the use of these lifesaving devices, new technologies have recently been developed, such as emergency dispatcher telephone-assisted AED localisation and mobile applications (1517). No consensus currently exists regarding a viable and realistic distance threshold for defining "nearby PAEDs". This distance, defined as how far a typical bystander would likely travel to 
retrieve an AED, is postulated to range from 100 to 400 meters, depending on the time allowed for the bystander to perform the task $(18,19)$.

In this study, bystander PAED shock in a major metropolitan area was studied by modeling the probability of its use according to multiple variables such as distance-to-PAED, delay of prehospital medical care arrival, and OHCA time of day and type of location (out-ofhome versus at-home).

\section{Methods}

\section{Study Design}

This study is a retrospective, observational, cross-sectional analysis. Emergency medical services (EMS) records were reviewed to identify all cases for which paramedics were dispatched for a non-traumatic OHCA between January $1^{\text {st }} 2014$ and December $31^{\text {st }} 2015$.

The urban community of greater Montreal (Quebec, Canada) is served by one EMS provider, Urgences-santé, with associated first responder agencies that are fire, police and volunteer based. For each cardiac arrest, first responders and paramedics are jointly dispatched to the scene by the regional communications central in order to minimize time to first shock.

Urgences-santé covers a 744- $\mathrm{km}^{2}$ territory with 2.4 million inhabitants and conducts 230,000 interventions per year. Over 800 paramedics are active in the system, with the overwhelming majority (99\%) of providers being primary care paramedics trained to the BLS level and employing semi-automatic defibrillators (20). Ten ACLS-trained advanced care paramedics are also active in the system (21).

For 2014-2015, Urgences-santé's reported Utstein criteria were as follows: the average 
OHCA patient age was 68.76 (standard deviation=18.25) and $61.4 \%$ of victims were male. Seventy-two percent of OHCA were witnessed by a bystander. Of these witnessed OHCA, 48\% received bystander $\mathrm{CPR}, 45 \%$ had an initial rhythm of pulseless ventricular tachycardia or ventricular fibrillation, $10 \%$ had an initial rhythm of asystolie, and 55\% had a prehospital return of spontaneous circulation (ROSC). Sixty-one percent of victims with prehospital ROSC survived to hospital discharge.

PAED deployment and accessibility in Montreal and Laval are not subject to any federal, provincial or municipal legislation. Therefore, AED placement in both public spaces (e.g. librairies, community centres, town halls) as well as private locations (e.g. business offices, clubs) has been uncoordinated. Moreover, registration of these devices with Urgences-santé and its communications central is on a voluntary basis.

Currently, Urgences-santé's dispatching software notifies emergency dispatchers managing an OHCA call of the presence of a PAED when the caller gives an address that is an exact match to an address in the PAED registry. Therefore, dispatchers only direct bystanders to a nearby PAED if there is a defibrillator in the building where the victim is located, when such information is known.

\section{Ethics Review}

The study's research protocol was approved by the Hôpital du Sacré-Coeur de Montréal clinical research ethics committee.

\section{Study Population and Data Collection}

Were included all adult (age $\geq 18$ ) prehospital victims who suffered a non-traumatic OHCA 
between January $1^{\text {st }} 2014$ and December $31^{\text {st }} 2015$ inclusively. All victims for whom the OHCA onset was witnessed by an on-duty EMS paramedic were excluded from the study.

Two full-time primary care paramedics with a minimum of 5 years of quality assurance experience at the time of the study extracted prehospital call information from the EMS electronic database, which contains information scanned in from manually entered prehospital run records. This database was electronically searchable for desired information. Information on incident time, location, first responder and EMS times of arrival, bystander AED shock and time to first EMS shock were collected for each OHCA. Records did not allow researchers to identify, among all OHCA not associated with a bystander shock, the OHCA where a bystander applied an AED but no defibrillation shock was advised.

For the studied territory, PAED locations were derived from a database containing all PAEDs currently registered at the EMS's communications central (487 devices at 187 distinct locations). The database was last fully updated in the spring of 2016.

\section{Measures}

Bystander shock was taken to be any case for which an AED operated by a "lay" (non-EMS) witness delivered at least one shock to the victim.

Distance between victim and registered PAED: this variable was calculated using a straightline distance between high-precision geographic coordinates of the arrival location of the ambulance (based on the vehicle's GPS system) and the street address of the building housing the PAED (based on geocoding using Google Maps with manual correction), projected using the UTM18 North coordinate reference system. 
At-home cardiac arrest was taken to be any case identified by the researchers where a patient was treated in a residence, which could include private houses or apartments, assisted living facilities and group homes.

Minimum response time was taken to be, in minutes, the minimum time any emergency responder took to arrive on scene. Responders could include fire-fighter first responders, municipal volunteer first responders, primary care paramedics, advanced care paramedics and AED-equipped police officers.

Business hours were defined as Monday to Friday, from 9 A.M. to 5 P.M. Non-weekend statuatory holidays were not excluded. The time-of-day variable (business hours versus evenings/night/week-ends) was a proxy to estimate both the likelihood of bystanders witnessing the arrest and the hours of availability of the PAEDs.

Analysis

Descriptive statistics (means and standard deviations/proportions) were employed to describe the study population's demographic and clinical characteristics. A chi-square test was used to evaluate differences between victims shocked and not shocked by a bystander. To visualize geographic patterns in the data, PAEDs and OHCAs were mapped by distance, location type, business hours and response time.

Multivariable logistic regressions were used to estimate the association between bystander shock and distance to nearest registered PAED (logged meters) for different arrest locations (stratified to at-home versus out-of-home), while controlling for time of day (dichotomized to office hours during the week versus evenings/nights/week-ends), and minimum response time (minutes). All results are reported using 95\% confidence intervals. 
All statistical analysis and geographic measurement was performed using the $\mathrm{R}$ language (version 3.3.0, The R Foundation for Statistical Computation, Vienna, Austria) with the sp package (version 1.2-3, Pebesma, E.J., R.S. Bivand, 2005. Classes and methods for spatial data in R. R News 5 (2), https://cran.r-project.org/doc/Rnews/). Cartography was performed using QGIS (version 2.18.0, QGIS Development Team, 2016. QGIS Geographic Information System. Open Source Geospatial Foundation. URL http://qgis.osgeo.org).

\section{Results}

\section{OHCA Characteristics}

Over a 24-month period, 2443 OHCA cases were identified. Regardless of victim distance-toPAED, a total of 77 OHCA cases were associated with bystander shock (3.2\%) versus 2366 cases without bystander shock (96.9\%). OHCA occurred at home in 1821 cases $(74.5 \%)$ versus in the public in 622 cases $(25.5 \%)$. Seven hundred fourty three patients $(30.4 \%)$ arrested during office hours.

Table 1 outlines the prehospital characteristics of OHCA victims associated with bystander shock and victims not associated with bystander shock. Results were not statistically different between the two groups with regards to response time for professional first responders (excluding EMS), reponse time for EMS primary care paramedics, response time for EMS advanced care paramedics and time to first EMS shock. However, minimum response time was statistically longer in the PAED bystander shock victim group. Moreover, professional first responder services arrived at the victim's bedside before EMS paramedics in $57.1 \%$ of cases where a PAED shock was delivered versus $80.3 \%$ of cases where no PAED was deliverered $(\mathrm{p}<0.01)$. The proportion of victims arresting out-of-home was greater among PAED-associated 
OHCA than those not associated with PAED-shock ( $58 \%$ vs $24 \%, \mathrm{p}<0.01)$. Victims shocked by a bystander arrested at a higher frequency during business hours than victims not shocked by a bystander $(42 \%$ vs $30 \%, \mathrm{p}=0.04)$.

\section{Table 1. OHCA victim characteristics}

\section{(bystander shock versus no bystander shock)}

Figure 1 shows noticeable spatial trends in response times. Delays are shortest in the densest neighbourhoods of Montreal and longer in the north and the West Island areas. Notably, Laval which does not have a fire service-based first responder program - suffers from the longest delays.

Figure 1. Map showing the location of registered PAED and

\section{OHCA cases with their response times}

Proximity to a PAED

The number of OHCA victims located within different distance-to-PAED thresholds were the following: 37 victims (1.5\%) were within 100 meters of a registered PAED, 118 (4.8\%) were within 200 meters, $240(9.8 \%)$ were within 300 meters and $373(15.3 \%)$ were within 400 meters. As can be seen in Figure 2, PAEDs closest to OHCA were clustered in the downtown and central areas. PAEDs in the West Island area and on Laval Island were located relatively far from any OHCA during the study period. These spatial patterns largely reflect population density across the two islands.

Figure 2. Map showing PAED by distance to nearest OHCA 
Figures $3 \mathrm{~A}$ and $3 \mathrm{~B}$ demonstrate the distribution of distance-to-PAED for victims who received a bystander shock versus those who did not, respectively.

Figure 3A. Distance-to-PAED histogram for bystander-shock victims

Figure 3B. Distance-to-PAED histogram for no bystander-shock victims

Logistic Regression Predicting Bystander Shock

Results from logistic regression models addressing PAED-use probability are described in Table 2.

\section{Table 2. Results of logistic regressions on data stratified by location of arrest}

Adjusted Odds Ratio for PAED-Shock versus Distance-to-PAED

Figure 4 is a plot of adjusted odds ratios resulting from logistic models regressing out-of-home PAED-shock against distance-to-PAED dichotimised (nearer/father) using a range of thresholds, while controlling for business hours and response time. The solid line shows adjusted odd ratios (aOR) for thresholds ranging from 25 to 600 meters; the dashed lines show the $95 \%$ confidence band; the red line shows $\mathrm{aOR}=1$. For thresholds up to 175 meters inclusively, the associations were statistically significant at alpha $=0.05$. The corresponding results for Figure 4 are tabulated in Table 3.

Figure 4. Plot of adjusted odds ratio for bystander shock according to distance-to-PAED thresholds for out-of-home OHCA

Table 3. Adjusted odds ratio for bystander shock according to distance-to-PAED thresholds for out-of-home OHCA 


\section{Discussion}

This study's results show that bystander shock prior to arrival of emergency prehospital care was a rare event in the Montreal area (3\% of all study cases) and lower than previously reported for other Canadian cities (14).

These findings shed light on factors that may influence bystander shock for OHCA resuscitation in the greater Montreal area and possibly other major urban centres.

First, the association between distance-to-PAED and bystander shock appears to be conditional on the type of location of the OHCA. Analysis showed a negative, marginally statistically significant association for out-of-home cardiac arrests (aOR=0.80, CI: 0.64-0.99), yet a positive non-significant association for at-home arrests $(\mathrm{aOR}=1.30, \mathrm{CI}$ : $0.83-2.05)$. If an arrest is not witnessed or an AED not deployed in the first few minutes the onset, the likelihood of bystander shock will be very low. Previous estimates suggest that PAEDs located 100 meters or less in distance to the bystander are reasonably near and can be retrieved within less than 3 minutes $(18,19)$. This study's results (Figure 4 and Table 3) demonstrate that out-of-hospital victims located within 100 meters of distance to a registered PAED are 4.81 times more likely to receive bystander shock than victims located more than 100 meters from a PAED (CI: 1.84 12.60).

In interpreting the results associating bystander shock odds to distance-to-PAED for outof-home cardiac arrest, the authors recognise two competing explanations. The observed distance decay in the odds of bystander shock (Figure 3) could reflect the distance at which PAEDs are used: a bystander is more likely to use a AED if one is closer to hand. In this respect, this study's data suggests that a 175 -meter distance threshold $(\mathrm{aOR}=2.52, \mathrm{CI}: 1.07-5.89)$ could be a viable 
definition for "nearby PAED" and serve strategies to increase PAED-use by helping bystanders locate nearby defibrillators, e.g. dispatcher-assisted AED localization and mobile applications. Such information could also help public health officials develop AED spatiotemporal access strategies. In fact, a recent study led in Toronto (Ontario, Canada) ranked businesses and municipal locations according to the number of OHCA occurring within 100 meters of their locations and during their opening hours, and found that coffee shops and bank machines from the 5 largest Canadian banks occupied 8 of the top 10 spots. (22)

Alternatively, the observed distance decay could reflect the distance at which PAED - an unknown number of which are missing from the Urgences-santé PAED registry - cluster geographically. The association between distance-to-PAED and bystander shock could reflect little more than the probability that bystanders near registered PAEDs (not used) are more likely to be in locations that house unregistered PAEDs (used). If this is the case, the authors would expect that the marginal effect of installing new PAEDs will be limited.

Urgences-santé's dispatching software notifies emergency dispatchers managing an OHCA call of the presence of a PAED when the caller gives an address that is an exact match to an address in the PAED registry. In this study, only eight cases of OHCA that occurred at locations in the PAED registry (defined as OHCA location less than 10 meters from a location in the registry). Therefore, the authors suspect that this Urgences-santé protocol is not responsible for the association between bystander shock and proximity to a PAED outside of the building in which the OHCA occurred.

Second, OHCA occurring during business hours had higher odds of receiving a shock from a bystander, but the association was not statistically significant (at-home aOR $=1.73, \mathrm{CI}$ : 
0.84-3.57; out-of-home aOR $=1.12, \mathrm{CI}: 0.60-2.08$ ). Temporal access to PAED is complex and this study's proxy probably did not adequately reflect the actual device availabilities. Previous research found that one in five OHCA occur near an inaccessible PAED at the time of the incident (23).

Third, longer delay to arrival of the quickest emergency responder had a positive association with bystander shock, but the association was only marginally significant for at-home arrests (at-home aOR $=1.04, \mathrm{CI}: 1.00-1.07$; out-of-home aOR $=1.06, \mathrm{CI}: 0.97-1.16$ ). The authors speculate that longer emergency care delays translate into more time for bystanders to retrieve and deploy a defibrillator. In this sense, urban locations with longer EMS arrival delays may benefit even more from greater defibrillator accessibility and density, whereas areas immediately surrounding fire-fighter stations may not require dense PAED accessibility.

In preparing this study, the authors did not aim to establish causation between PAED bystander shock and OHCA survival since this as been well established in prior trials $(5,8,10$, 11).

\section{Limitations}

This study has a number of limitations. First, as a retrospective observational study, results are subject to confounding. Indeed, as explained prior, the significant association discovered between bystander PAED-shock and distance-to-PAED can be interpreted in two divergent ways. Furthermore, the 175-meter distance-to-PAED threshold obtained in this study may likely increase in the future if dispatcher-assisted AED localization and mobile apps become more widespread. 
Second, study data did not provide information on the source of the AED used to shock the victim. Figure 3A demonstrates the amplitude of this limitation: the distance-to-PAED for certain bystander-shock victims largely surpasses viable distances one can expect a bystander to travel in order to recuperate and use an AED prior to EMS arrival. This lack of discrimination between cases where the AED employed was the closest registered PAED and cases where it was not limits the interpretability and generalisability of the findings.

Third, the level of precision in the coding of location type did not allow researchers to distinguish between cases of OHCA in private homes (where the rate of on-site AED is expected to be very low) and those in assisted-living situations (where the rate of on-site AED is expected to be very high). This lack of discrimination also limits the interpretability and generalisability of the findings.

Fourth, the study did not address OHCA cases where an AED was deployed by a bystander, but no shock was advised or the bystander failed to deliver an advised shock. This may limit the generalisability of the results.

Fifth, the PAED registry used in this study was last updated in spring 2016, but it was compared with OHCA having occurred in 2014 and 2015. As PAED locations and temporal availability are continuously changing, it is conceivable that the calculations mismatched the closest PAEDs for some OHCA. The authors suspect that PAEDs are increasingly widespread. Therefore, such a mismatch may have biased the results towards the null hypothesis and the association may in fact be stronger than the results suggest.

Last, a recent study conducted in Toronto (Ontario, Canada) demonstrated that one in five OHCA occur near an inaccessible PAED at the time of the incident (23). In this study, 
researchers did not have access to each individual PAED's schedule of availability and therefore assumed them to be available at all times, while including standard office hours as a proxy for availability in the regression. Again, this limitation may have biased the results towards the null hypothesis.

\section{Conclusion}

Bystander shock occured in 3\% of non-traumatic out-of-hospital cardiac arrests in the greater Montreal area. The closer a victim of out-of-home cardiac arrest was to a registered public automated external defibrillator, the higher the odds that they received a bystander shock. Outof-home victims who arrested 175 meters or less from a registered PAED had significantly higher odds of receiving bystander shock, regardless of OHCA onset time and emergency medical services arrival delays.

\section{Author Contributions}

JNB: designed and conceptualized the study, performed analysis and interpretation of results, and drafted the manuscript.

LdeM: assisted in designing and conceptualizing the study, performed analysis and interpretation of results, created the maps and plots, and revised the manuscript.

DR: assisted in the interpretation of results, and revised the manuscript.

FdeC: assisted in the designing and conceptualizing the study and revised the manuscript.

ES: assisted in designing and conceptualizing the study, obtained clinical research ethics committee approval, assisted with interpretation of results, and revised the manuscript. 


\section{Study Funding}

Urgences-santé research personnel were remunerated for their work on this study. Other authors did not receive funding.

\section{Disclosure}

FdeC is President of the Jacques-de Champlain Foundation, a non-profit organization whose mission is to promote cardiovascular research and to improve resuscitation care in the province of Quebec. JNB was commissionned by the Jacques-de Champlain Foundation from June 2016 to May 2017 to populate the first provincial public automated external defibrillator registry in Quebec. Other authors report no disclosures.

\section{References}

1. Daya MR, Schmicker RH, Zive DM, Rea TD, Nichol G, Buick JE, et al. Out-of-hospital cardiac arrest survival improving over time: Results from the Resuscitation Outcomes Consortium (ROC). Resuscitation. 2015;91:108-15.

2. Mozaffarian D, Benjamin EJ, Go AS, Arnett DK, Blaha MJ, Cushman M, et al. Heart Disease and Stroke Statistics-2016 Update: A Report From the American Heart Association. Circulation. 2016;133(4):e38-360.

3. Robertson RM. Sudden death from cardiac arrest - improving the odds. N Engl J Med. 2000;343(17):1259-60.

4. Weisfeldt ML, Sitlani CM, Ornato JP, Rea T, Aufderheide TP, Davis D, et al. Survival after application of automatic external defibrillators before arrival of the emergency medical system: evaluation in the resuscitation outcomes consortium population of 21 million. J Am Coll Cardiol. 2010;55(16):1713-20.

5. Berdowski J, Blom MT, Bardai A, Tan HL, Tijssen JG, Koster RW. Impact of onsite or dispatched automated external defibrillator use on survival after out-of-hospital cardiac arrest. Circulation. 2011;124(20):2225-32.

6. Morrison LJ, Verbeek PR, Vermeulen MJ, Kiss A, Allan KS, Nesbitt L, et al. Derivation and evaluation of a termination of resuscitation clinical prediction rule for advanced life support providers. Resuscitation. 2007;74(2):266-75.

7. Sasson C, Hegg AJ, Macy M, Park A, Kellermann AL, McNally B. Prehospital termination of resuscitation in cases of refractory out-of-hospital cardiac arrest. JAMA. 2008;300(12):1432-8. 
8. Sasson C, Rogers MA, Dahl J, Kellermann AL. Predictors of survival from out-ofhospital cardiac arrest: a systematic review and meta-analysis. Circ Cardiovasc Qual Outcomes. 2010;3(1):63-81.

9. Drennan IR, Lin S, Thorpe KE, Morrison LJ. The effect of time to defibrillation and targeted temperature management on functional survival after out-of-hospital cardiac arrest. Resuscitation. 2014;85(11):1623-8.

10. Larsen MP, Eisenberg MS, Cummins RO, Hallstrom A. Predicting survival from out-ofhospital cardiac arrest: a graphic model. Ann Emerg Med. 1992;22(22):1652-8.

11. Sanna T, La Torre G, de Waure C, Scapigliati A, Ricciardi W, Dello Russo A, et al. Cardiopulmonary resuscitation alone vs. cardiopulmonary resuscitation plus automated external defibrillator use by non-healthcare professionals: a meta-analysis on 1583 cases of out-ofhospital cardiac arrest. Resuscitation. 2008;76(2):226-32.

12. Weisfeldt ML, Everson-Stewart S, Sitlani CM, Rea T, Aufderheide TP, Atkins D, et al. Ventricular tachyarrhythmias after cardiac arrest in public versus at home. N Engl J Med. 2011;364:313-21.

13. Capucci A, Aschieri D, Guerra F, Pelizzoni V, Nani S, Villani GQ, et al. Communitybased automated external defibrillator only resuscitation for out-of-hospital cardiac arrest patients. Am Heart J. 2016;172:192-200.

14. Brooks SC, Lam KK, Morrison LJ. Out-of-hospital cardiac arrests occuring in southern Ontario health care clinics: bystander cardiopulmonary resuscitation and automated external defibrillator use. Can Fam Physician. 2010;56:e213-18.

15. Brooks SC, Simmons G, Worthington H, Bobrow BJ, Morrison LJ. The PulsePoint Respond mobile device application to crowdsource basic life support for patients with out-ofhospital cardiac arrest: Challenges for optimal implementation. Resuscitation. 2016;98:20-6.

16. Ringh M, Rosenqvist M, Hollenberg J, Jonsson M, Fredman D, Nordberg P, et al. Mobile-phone dispatch of laypersons for CPR in out-of-hospital cardiac arrest. N Engl J Med. 2015;372(24):2316-25.

17. Riyapan S, Lubin J. Emergency dispatcher assistance decreases time to defibrillation in a public venue: a randomized controlled trial. Am J Emerg Med. 2016;34(3):590-3.

18. Bohannon RW. Comfortable and maximum walking speed of adults aged 20-79 years: reference values and determinants. Age and Ageing. 1997;26:15-9.

19. Hazinski MF, Idris AH, Kerber RE, Epstein A, Atkins D, Tang W, et al. Lay rescuer automated external defibrillator ("public access defibrillation") programs: lessons learned from an international multicenter trial: advisory statement from the American Heart Association Emergency Cardiovascular Committee; the Council on Cardiopulmonary, Perioperative, and Critical Care; and the Council on Clinical Cardiology. Circulation. 2005;111(24):3336-40. 20. Berg RA, Hemphill R, Abella BS, Aufderheide TP, Cave DM, Hazinski MF, et al. Adult Basic Life Support: 2010 American Heart Association Guidelines for Cardiopulmonary Resuscitation and Emergency Cardiovascular Care. Circulation. 2010;122(18 Suppl 3):S685705 .

21. Neumar RW, Otto CW, Link MS, Kronick SL, Shuster M, Callaway CW, et al. Adult Advanced Cardiovascular Life Support: 2010 American Heart Association Guidelines for Cardiopulmonary Resuscitation and Emergency Cardiovascular Care. Circulation. 2010;122(18 Suppl 3):S729-67. 
22. Sun CL, Brooks S, Morrison LJ, Chan TC. Ranking Businesses and Municipal Locations by Spatiotemporal Cardiac Arrest Risk to Guide Public Defibrillator Placement. Circulation. 2017;135(12):1104-20.

23. Sun CL, Demirtas D, Brooks SC, Morrison LJ, Chan TC. Overcoming Spatial and Temporal Barriers to Public Access Defibrillators Via Optimization. J Am Coll Cardiol. 2016;68(8):836-45. 


\section{Tables}

Table 1. OHCA victim characteristics (bystander shock versus no bystander shock)

Characteristic
Bystander shock No bystander shock p-value

$$
\begin{array}{ll}
(\mathrm{N}=77) & (\mathrm{N}=2366) \\
\text { mean }(\mathrm{SD} \text { or } \%) & \text { mean }(\mathrm{SD} \text { or \%) }
\end{array}
$$

\begin{tabular}{lccc}
\hline Delays & & & \\
Responder time for professional first & $5.9(2.2)$ & $5.9(3.2)$ & 0.95 \\
responders excluding EMS (minutes) & & & \\
Reponse time for EMS primary care & $8.7(4.4)$ & $8.8(4.9)$ & 0.77 \\
paramedics (minutes) & & & 0.32 \\
Reponse time for EMS advanced care & $17.5(6.5)$ & $16.1(7.0)$ & $0.03 *$ \\
paramedics (minutes) & & & 0.71 \\
Minimum response time (minutes) & $7.3(4.3)$ & $14.0(7.3)$ & $0.7)$ \\
Time to first EMS shock (minutes) & $13.1(7.3)$ &
\end{tabular}

\section{Location}

Out of home

$45(58.4 \%)$

$576(24.3 \%)$

$<0.01 *$

\section{Time of day}

Business hours

$32(41.6 \%)$

$711(30.1 \%)$

0.04 * 
Note: minimum response time corresponds to the minimum time any emergency responder took to arrive on scene (either fire fighter first responders, municipal volunteer first responders, AEDequipped police officers, EMS primary care paramedics or EMS advanced care paramedics). The fact that not all victims benefited from the presence of professional first responders before the arrival of EMS explains why the average minimum response times are in fact longer than the average response times for professional first responders. 
Table 2. Results of logistic regressions on data stratified by location of arrest

\begin{tabular}{lcc} 
At-home OHCA & aOR & CI (95\%) \\
\hline \hline Distance to PAED (logged m) & 1.30 & {$[0.83-2.05]$} \\
Response time (minutes) & 1.04 & {$[1.00-1.07]$} \\
Business hours (ref: during hours) & 1.73 & {$[0.84-3.57]$} \\
& & \\
Out-of-home OHCA & aOR & CI (95\%) \\
& & \\
\hline \hline Distance to PAED (logged m) & 0.80 & {$[0.64-0.99]$} \\
& & \\
Response time (minutes) & 1.06 & {$[0.97-1.16]$} \\
Business hours (ref: during hours) & 1.12 & {$[0.60-2.08]$}
\end{tabular}

Note: $\mathrm{aOR}=$ adjusted odds ratio; $\mathrm{CI}=$ confidence interval 
Table 3. Adjusted odds ratio for bystander shock according to distance-to-PAED thresholds for out-of-home OHCA

\begin{tabular}{|c|c|c|}
\hline $\begin{array}{l}\text { Distance-to-PAED } \\
\text { threshold }\end{array}$ & aOR & CI (95\%) \\
\hline 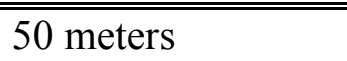 & 9.13 & 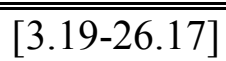 \\
\hline 100 meters & 4.82 & [1.84-12.60] \\
\hline 150 meters & 3.02 & {$[1.28-7.13]$} \\
\hline 200 meters & 2.15 & {$[0.93-5.00]$} \\
\hline 250 meters & 1.88 & {$[0.86-4.13]$} \\
\hline 300 meters & 1.41 & {$[0.65-3.03]$} \\
\hline 350 meters & 1.70 & {$[0.85-3.38]$} \\
\hline 400 meters & 1.93 & [1.01-3.68] \\
\hline 450 meters & 1.42 & {$[0.75-2.71]$} \\
\hline 500 meters & 1.40 & {$[0.74-2.63]$} \\
\hline 550 meters & 1.18 & {$[0.63-2.22]$} \\
\hline 600 meters & 1.08 & {$[0.58-2.01]$} \\
\hline
\end{tabular}




\section{Figures}

Figure 1. Map showing the location of registered PAED and OHCA cases with their response times

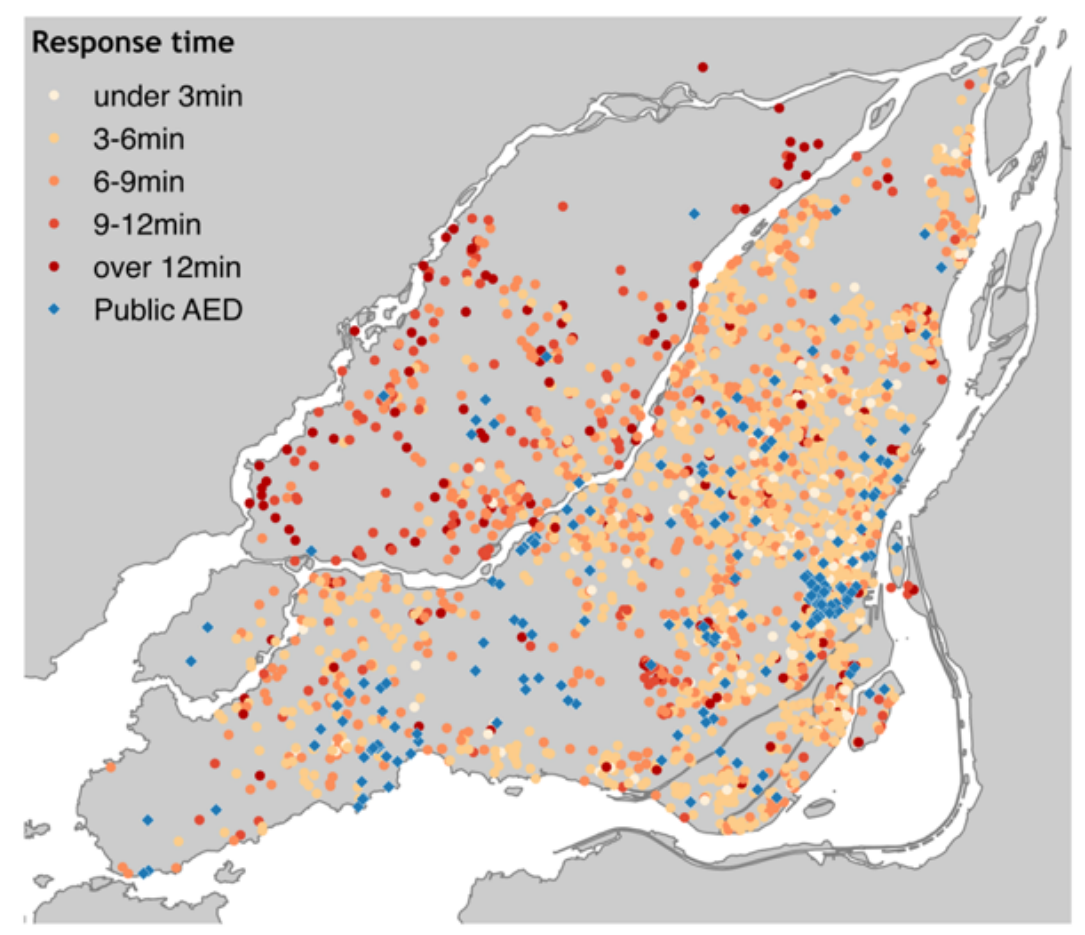


Figure 2. Map showing PAED by distance to nearest OHCA

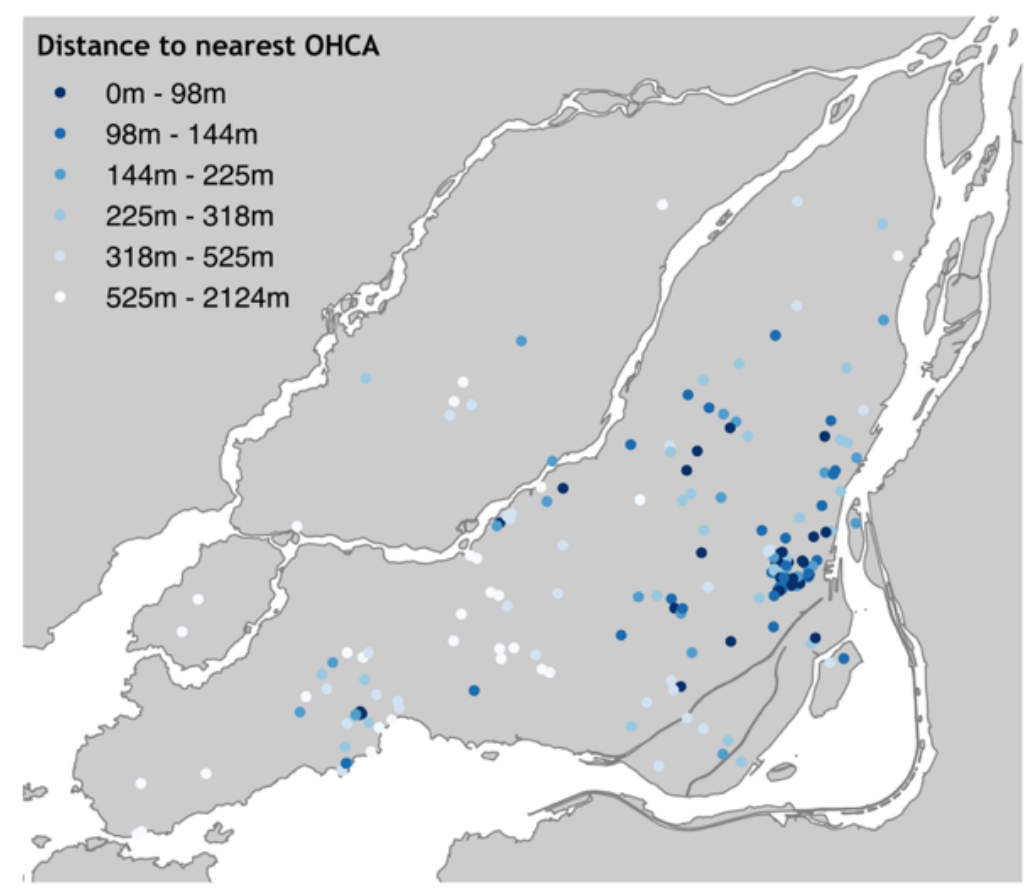


Figure 3A. Distance-to-PAED histogram for bystander-shock victims

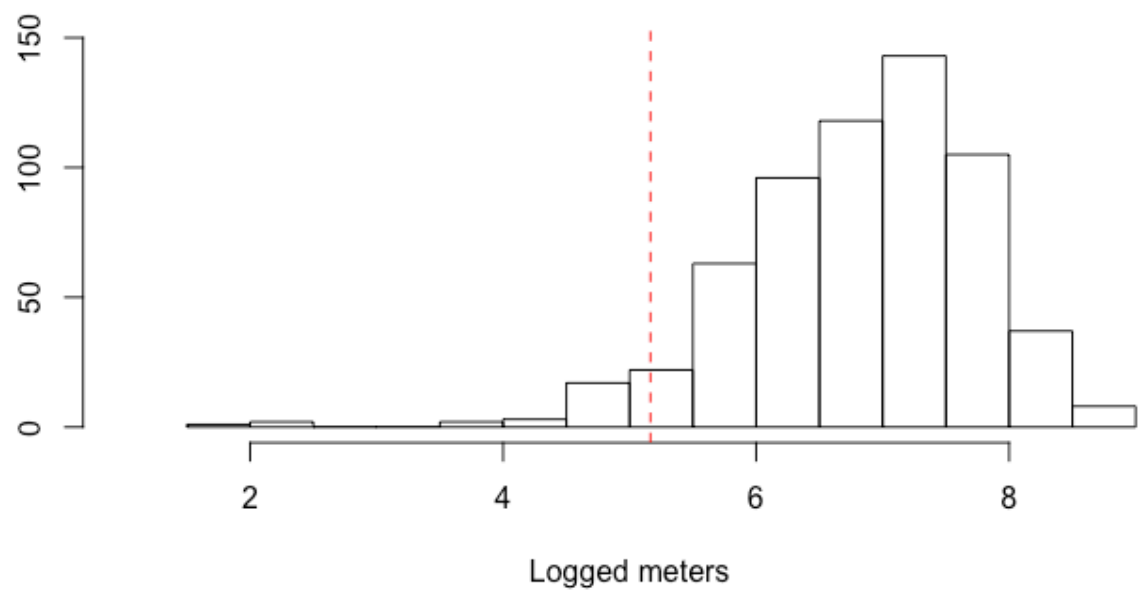

Figure 3B. Distance-to-PAED histogram for no bystander-shock victims

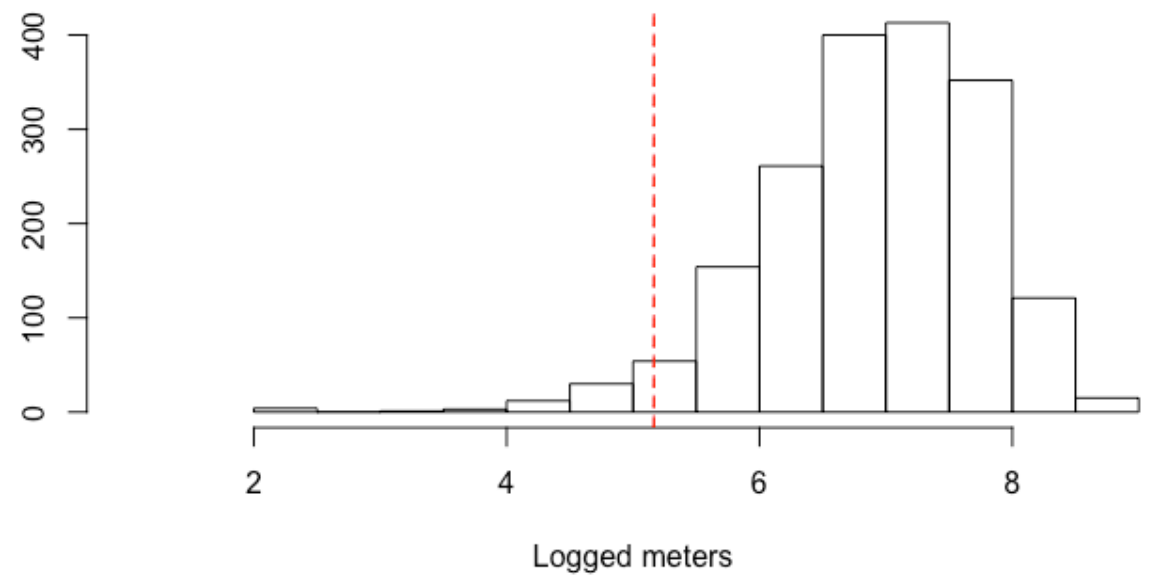


Figure 4. Plot of adjusted odds ratio for bystander shock according to distance-to-PAED thresholds for out-of-home OHCA

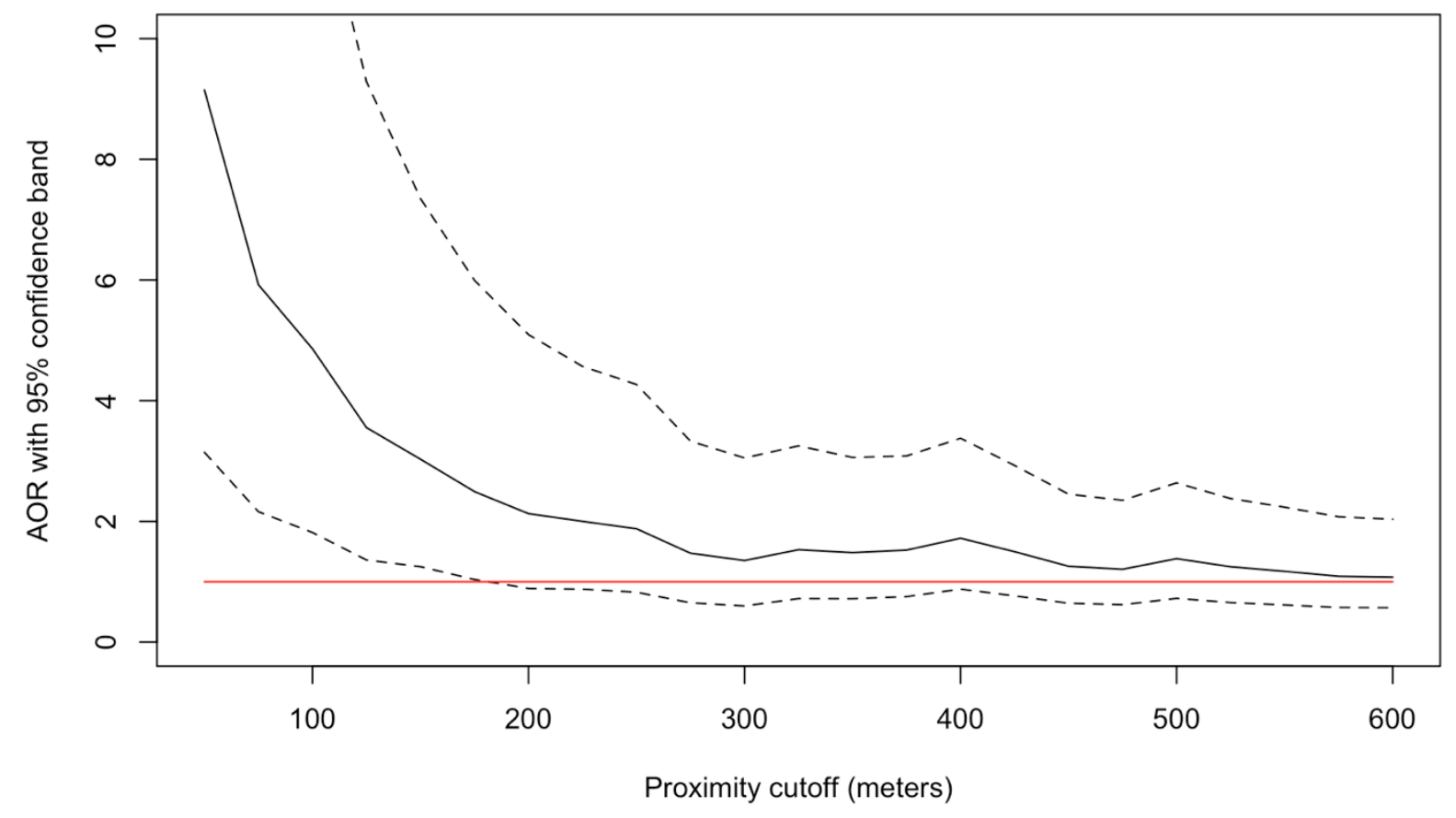

